\title{
Towards a standard completeness for a probabilistic logic on infinite-valued events
}

\author{
Tommaso Flaminio \\ Artificial Intelligence Research Institute (IIIA - CSIC) \\ Campus de la Univ. Autònoma de Barcelona s/n, 08193 Bellaterra, Spain. \\ tommaso@iiia.csic.es
}

\begin{abstract}
MV-algebras with internal states, or SMV-algebras for short, are the equivalent algebraic semantics of the logic $\operatorname{SFP}(\mathrm{E}, \mathrm{E})$ which allows to represent and reason about the probability of infinite-valued events. In this paper we will make the first steps towards establishing completeness for $\mathrm{SFP}(\mathrm{E}, \mathrm{E})$ with respect to the class of standard SMV-algebras, a problem which has been left open since the first paper on SMV-algebras was published. More precisely we will prove that, if we restrict our attention to a particular, yet expressive, subclass of formulas, then theorems of $\operatorname{SFP}(\mathrm{E}, \mathrm{E})$ are the same as tautologies of a class of SMV-algebras that can be reasonably called "standard".
\end{abstract}

Keywords. MV-algebras, states, internal states, standard completeness, probabilistic logic.

\section{Introduction}

MV-algebras with an internal state, or SMV-algebras, have been introduced in [6] and they consist of an MV-algebra A (cf. [2]) and a unary map $\sigma$ on $\mathbf{A}$ axiomatized so as to preserve some basic properties of a state of $\mathbf{A}$ (cf. [12,5]), i.e., a $[0,1]$-valued, normalized and finitely additive function. These structures form a variety, denoted by $\mathbb{S M V}$, which is the equivalent algebraic semantics for the probabilistic logic $\mathrm{SFP}(\mathrm{E}, \mathrm{E})$ which permits to represent and reason about the probability of infinite-valued events. In that logic, in particular, events are described by formulas of the infinite-valued Łukasiewicz calculus (cf. [2]).

From the perspective of reasoning about uncertainty, the interest of Eukasiewicz events is twofold: whilst capturing properties of the world which are better described as gradual rather than yes-or-no, they also mimic bounded random variables. Indeed, any Łukasiewicz event $e$ may be regarded as a $[0,1]$-valued continuous function $e^{*}$ on a compact Hausdorff space (see [2, Theorem 9.1.5] and Section 2) and any state of $e$ coincides with the expected value of $e^{*}$ (see [4, Remark 2.8] and Section 3).

SMV-algebras provide a framework to treat a generalization of classical probability theory in a universal-algebraic setting. However, notwithstanding their meaningful expressive power, several problem concerning their universal-algebraic properties are still open. Here, we will concentrate on the following issue: determining a proper subclass of SMV-algebras which generates $\mathbb{S M V}$ and which 
could be regarded as a class of "measure-theoretical" models. In other words, the above problem is to establishing a "standard completeness" for the logic $\operatorname{SFP}(\mathrm{E}, \mathrm{E})$.

In particular we will show that, upon restricting our language to a special, yet sufficiently expressive subset of well-formed formulas, the logic $\operatorname{SFP}(\mathrm{E}, \mathrm{E})$ turns out to be sound and complete with respect to a prominent subclass of SMV-algebras which can be reasonably called "standard". Indeed, these standard $\mathrm{SMV}$-algebras are grounded on the same MV-algebra of continuous real-valued functions and their internal states are obtained by integrating the continuous functions which correspond to Lukasiewicz events by a regular, Borel (and hence $\sigma$-additive) probability measure. Although our main result only offers a partial solution to the problem of establishing standard completeness for $\operatorname{SFP}(\mathrm{E}, \mathrm{E})$, the idea behind its proof is promising and it suggests ways to tackle the general problem. Indeed, the class of formulas to which we will restrict our attention, called purely probabilistic formulas, form the ground probabilistic language upon which the all formulas of $\mathrm{SFP}(\mathrm{E}, \mathrm{E})$ are inductively defined.

This paper is organizes as follows: in the next section we will recall some basic definitions and needed results about Lukasiewicz logic and MV-algebras. Section 3 is dedicated to a brief introduction to generalized probability theory on Lukasiewicz logic, i.e., state theory, while in Section 4 we will focus on SMValgebras and the probabilistic logic $\operatorname{SFP}(\mathrm{E}, \mathrm{E})$. In Section 5 we will prove the main results of this paper and in particular that a purely probabilistic formula $\phi$ is a theorem of $\operatorname{SFP}(\mathrm{L}, \mathrm{L})$ iff $\phi$ holds in all standard SMV-algebras.

\section{2 Łukasiewicz logic and MV-algebras}

The language of the infinite-valued Łukasiewicz calculus $\mathrm{E}$ is made of a countable set of propositional variables $\left\{q_{1}, q_{2}, \ldots\right\}$, the binary connective $\oplus$, the unary connective $\neg$ and the constant $\perp$. Formulas are defined by the usual inductive rules. Further useful connectives are definable as follows:

$$
\begin{aligned}
& \top=\neg \perp ; \varphi \odot \psi=\neg(\neg \varphi \oplus \neg \psi) ; \varphi \rightarrow \psi=\neg \varphi \oplus \psi ; \varphi \wedge \psi=\varphi \odot(\varphi \rightarrow \psi) ; \\
& \varphi \vee \psi=\neg(\neg \varphi \wedge \neg \psi) ; \varphi \ominus \psi=\neg(\varphi \rightarrow \psi) ; d(\varphi, \psi)=(\varphi \rightarrow \psi) \oplus(\psi \rightarrow \varphi) .
\end{aligned}
$$

We invite the reader to consult $[8,2]$ for an axiomatization of Lukasiewicz logic. For the sake of the present paper it is important to recall that this calculus has an equivalent algebraic semantics: the variety $\mathbb{M V}$ of $M V$-algebras. These are structures of the form $\mathbf{A}=(A, \oplus, \neg, \perp$ ) (the same language of Eukasiewicz logic), where $(A, \oplus, \perp)$ is a commutative monoid and, defining further operations as above, the following equations hold:

$$
\begin{aligned}
& x \oplus \top=\top \\
& (x \rightarrow y) \rightarrow y=(y \rightarrow x) \rightarrow x .
\end{aligned}
$$

The algebraizability of Eukasiewicz logic with respect to $\mathbb{M V}$ implies that $\mathrm{E}$ is sound and complete w.r.t. the class of MV-algebras. This means the following: for every MV-algebra $\mathbf{A}$, define an $\mathbf{A}$-valuation as a map $v$ from the propositional 
variables $q_{i}$ 's to $A$ which extends to all formulas by truth functionality; then, a formula $\varphi$ is a theorem of Eukasiewicz logic iff for every MV-algebra $\mathbf{A}$ and every A-valuation $v, v(\varphi)=\top$.

Furthermore, every formula $\varphi$ on propositional variables $q_{1}, \ldots, q_{k}$ can be regarded, on the algebraic side, as a term $t\left(x_{1}, \ldots, x_{k}\right)$ on the same number of variables. For every MV-algebra $\mathbf{A}$ and for every term $t$, we denote by $t^{\mathbf{A}}$ the interpretation (or semantics) of $t$ in $\mathbf{A}$.

In every MV-algebra the relation $x \leq y$ iff $x \rightarrow y=\top$ determines a latticeorder which coincides with the one given by the operations $\wedge$ and $\vee$. If in $\mathbf{A}$ the order $\leq$ is linear, we will say that $\mathbf{A}$ is an $M V$-chain.

The following collects two typical examples of MV-algebras which will play a central role in this paper.

Example 1. (1) The standard MV-algebra $[0,1]_{M V}$ has support on the real unit interval $[0,1]$ and for all $x, y \in[0,1], x \oplus y=\min \{1, x+y\}, \neg x=1-x$ and $\perp=0$. The semantics, in $[0,1]_{M V}$, of the Lukasiewicz connectives we defined above is as follows:

$$
\begin{gathered}
\top=1 ; x \odot y=\max \{0, x+y-1\} ; x \rightarrow y=\min \{1,1-x+y\} ; \\
x \wedge y=\min \{x, y\} ; x \vee y=\max \{x, y\} ; x \ominus y=\max \{0, x-y\} ; \\
d(x, y)=|x-y| \text { (the usual Euclidean distance). }
\end{gathered}
$$

Chang's theorem $[1]$ shows that $\mathbb{M V}$ is generated by $[0,1]_{M V}$, whence Eukasiewicz logic is complete w.r.t. to $[0,1]_{M V}$.

(2) For every $k \in \omega$, let $\mathcal{F}(k)$ be the set of functions $f:[0,1]^{k} \rightarrow[0,1]$ which are continuous, piecewise linear and such that each piece has integer coefficient, i.e., the set of $k$-variable McNaughton functions [2]. The point-by-point application of the operations of $[0,1]_{M V}$ makes $\mathcal{F}(k)$ an MV-algebra which coincides, up to isomorphism, with the free MV-algebra over $k$-variables. For every formula $\psi$ of Łukasiewicz language, we will denote by $f_{\psi}$ its corresponding McNaughton function. The free MV-algebra over infinitely-many generators will be henceforth denoted by $\mathcal{F}(\omega)$.

Let $\mathbf{A}$ and $\mathbf{B}$ be two MV-algebras. An MV-homomorphism is a function $h: A \rightarrow B$ such that, adopting without danger of confusion the same symbols for the operations of both algebras: (1) $h(\perp)=\perp ;(2) h(x \oplus y)=h(x) \oplus h(y) ;(3)$ $h(\neg x)=\neg h(x)$. If $S$ is a subset of $A$, a map $h: S \rightarrow B$ is a partial homomorphism provided that the above conditions (1-3) holds for the elements of $S$. Injective (partial) homomorphisms are called (partial) embeddings. (Partial) embeddings of $\mathbf{A}$ to $\mathbf{B}$ will be denoted by $\mathbf{A} \hookrightarrow \mathbf{B}$ ( $\mathbf{A} \hookrightarrow^{p} \mathbf{B}$, respectively).

Let $\mathbf{A}$ be an MV-algebra and let $S$ be a subset of $A$. We denote $\langle S\rangle_{\mathbf{A}}$ the MVsubalgebra of $\mathbf{A}$ generated by $S$. In particular, if $S$ is finite, for every $b \in\langle S\rangle_{\mathbf{A}}$, there are $a_{1}, \ldots, a_{n} \in S$ and an $n$-ary term $t$ such that $b=t^{\mathbf{A}}\left(a_{1}, \ldots, a_{n}\right)$.

Lemma 1. (1) For every $M V$-chain $\mathbf{A}$ and for every finite subset $S$ of $A$, there exists a partial embedding $h_{S}: S \hookrightarrow^{p}[0,1]_{M V}$.

(2) Let $\mathbf{A}, \mathbf{B}$ be $M V$-algebras, let $S$ be a finite subset of $A$. Every partial homomorphism $h_{S}: S \subseteq A \rightarrow B$ extends to a homomorphisms $\hat{h}_{S}:\langle S\rangle_{\mathbf{A}} \rightarrow \mathbf{B}$. 
Proof. (1) Immediately follows from Gurevich-Kokorin theorem (see for instance [8, Theorem 1.6.17]).

(2) For every element $b \in\langle S\rangle_{\mathbf{A}}$, there is an term $t$ and $a_{1}, \ldots, a_{n} \in S$ such that $b=t^{\mathbf{A}}\left(a_{1}, \ldots, a_{n}\right)$. Define $\hat{h}_{S}:\langle S\rangle_{\mathbf{A}} \rightarrow \mathbf{B}$ as follows:

$$
\hat{h}(b)=\hat{h}\left(t^{\mathbf{A}}\left(a_{1}, \ldots, a_{n}\right)\right)=t^{\mathbf{B}}\left(h\left(a_{1}\right), \ldots, h\left(a_{n}\right)\right) .
$$

It is easy to see that $\hat{h}$ commutes with the operations of $\mathbf{A}$ and $\mathbf{B}$, whence it is an MV-homomorphism.

An ideal of an MV-algebra $\mathbf{A}$ is a subset $I$ of $A$ such that: (1) $\perp \in I ;(2)$ if $a \in I$ and $b \in I$, then $a \oplus b \in I$; (3) if $a \in I$ and $b \leq a$, then $b \in I$. Every ideal $I$ of $\mathbf{A}$ determines a congruence $\Theta_{I}$ of $\mathbf{A}$ :

$$
(a, b) \in \Theta_{I} \text { iff } d(a, b) \in I .
$$

For every MV-algebra $\mathbf{A}$ and every ideal $I$ of $\mathbf{A}$, we denote by $\mathbf{A} / I$ the quotient of $\mathbf{A}$ by the congruence $\Theta_{I}$. Further, for every $a \in A$, we denote by $a / I$ the equivalence class of $a$ modulo $\Theta_{I}: a / I=\{b \in A \mid d(a, b) \in I\}$. The set of ideals of any MV-algebra, ordered by the set-theoretical inclusion, forms a lattice whose maximal elements are called maximal ideals. We write $\operatorname{Max}(\mathbf{A})$ for the set of maximal ideals of $\mathbf{A}$. The intersection of all maximal ideals of an MV-algebra $\mathbf{A}$ is an ideal, called the radical of $\mathbf{A}$ and denoted by $\operatorname{Rad}(\mathbf{A})$.

In the rest of this paper two kinds of structures will be of particular interest: simple and semisimple MV-algebras. The latter are characterized as those MVchains which, up to isomorphism, are MV-subalgebra of $[0,1]_{M V}$ (and therefore can be regarded as algebras of real numbers) [2, Theorem 3.5.1]; the former are algebras of continuous [0,1]-valued functions defined on a compact Hausdorff space [2, Corollary 3.6.8]. It is worth to recall that semisimple MV-algebras are those structures whose radical coincides with $\{\perp\}$. Moreover, for every MValgebra $\mathbf{A}$, the quotient $\mathbf{A} / \operatorname{Rad}(\mathbf{A})$ is semisimple (see [2, Lemma 3.6.6]) and it will be called the most general semisimple quotient of $\mathbf{A}$.

\section{Probability theory on Łukasiewicz events}

States of MV-algebras were introduced by Mundici in [12] as averaging values of Łukasiewicz truth-valuations.

Definition 1. A state of an $M V$-algebra $\mathbf{A}$ is a map $s: A \rightarrow[0,1]$ satisfying the following conditions:

(1) $s(\top)=1$,

(2) forall $x, y \in A$ such that $x \odot y=\perp, s(x \oplus y)=s(x)+s(y)$.

While condition (1) says that every state is normalized, (2) is usually called additivity with respect to Łukasiewicz sum $\oplus$. Indeed, the requirement $x \odot y=0$ is analogous to disjointness of a pair of elements in a Boolean algebra: if $\mathbf{A}$ is a 
Boolean algebra, then $x \odot y=\perp$ iff $x \wedge y=\perp$. Thus states generalize finitely additive probabilities to the realm of MV-algebras: finitely additive probabilities on a Boolean algebra are states as a special case of the above definition.

The following results collect properties of states which will be used in the proof of the main result of this paper.

Proposition 1 ([5, Proposition 3.1.7(ii)]). For every $M V$-algebra A, for every state $s$ of $\mathbf{A}$ and for every $a, b \in A$, if $a / \operatorname{Rad}(\mathbf{A})=b / \operatorname{Rad}(\mathbf{A})$, then $s(a)=s(b)$.

Proposition 1 above implies that every MV-algebra A and its most general semisimple quotient $\mathbf{A} / \operatorname{Rad}(\mathbf{A})$ have the same class of states. Thus, every state $s$ of any MV-algebra $\mathbf{A}$ assigns, for every $a, b \in A$ with $b \in a / \operatorname{Rad}(\mathbf{A})$, the same value $s(a) \in[0,1]$.

The following has been proved in [11, Theorem 6] and provides an MVanalogous of the well-known Horn-Tarski extension theorem [9].

Proposition 2. Let $\mathbf{A}$ and $\mathbf{B}$ be $M V$-algebras and let $\mathbf{B}$ be an $M V$-subalgebra of A. Every state $s_{B}: B \rightarrow[0,1]$ extends (not uniquely) to a state $s_{A}: A \rightarrow[0,1]$.

Remark 1. Every homomorphism $h$ of an MV-algebra $\mathbf{B}$ to $[0,1]_{M V}$ is a state. Thus, if $\mathbf{B}$ is an MV-subalgebra of $\mathbf{A}$, Proposition 2 shows that $h$ extends (not uniquely) to a state of $\mathbf{A}$. In other words, for every pairs of MV-algebras $\mathbf{B}$ and $\mathbf{A}$ such that $\mathbf{B}$ is an MV-subalgebra of $\mathbf{A}$ and for every homomorphism $h: \mathbf{B} \rightarrow$ $[0,1]_{M V}$, there exists a state $s$ of $\mathbf{A}$ such that for every $b \in B, s(b)=h(b)$.

The following theorem, independently proved by Kroupa [10] and Panti [14] (see also $[5, \S 4]$ ), represents every state $s$ of an MV-algebra $\mathbf{A}$ as the Lebesgue integral given by a unique regular, Borel probability measure. More precisely, every state of an MV-algebra $\mathbf{A}$ is the Lebesgue integral on the continuous functions $a^{*}$ 's of its most general semisimple quotient, defined on the compact Hausdorff space $\operatorname{Max}(\mathbf{A})$ of maximal ideals of $\mathbf{A}$.

Theorem 1. For every $M V$-algebra $\mathbf{A}$ and for every state $s$ of $\mathbf{A}$ there exists a unique regular, Borel probability measure $\mu$ on the Borel subsets of Max $(\mathbf{A})$ such that, for every $a \in A$,

$$
s(a)=\int_{\operatorname{Max}(\mathbf{A})} a^{*} \mathrm{~d} \mu
$$

\section{A probabilistic logic on Łukasiewicz events}

In this section we will recall basic definitions and properties for the logics $\mathrm{SFP}(\mathrm{E}, \mathrm{E})$ and its algebraic semantics ${ }^{1}$. The language of $\operatorname{SFP}(\mathrm{E}, \mathrm{E})$ is obtained by expanding that of Łukasiewicz infinite-valued calculus with a unary modality $P$. The set SPFm of well-formed formulas in this language is defined by

\footnotetext{
${ }^{1}$ We invite the reader to consult $[6,7]$ and $[5, \S 6]$ for a more exhaustive introduction to SMV-algebras, fuzzy probabilistic logics and their relation with uncertain reasoning.
} 


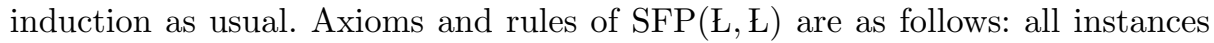
of axioms and rules of Łukasiewicz logic, plus the following axioms and rules for the modality $P$

- $P(\perp) \rightarrow \perp$ (Normalization);

- $P(\varphi \rightarrow \psi) \rightarrow(P(\varphi) \rightarrow P(\psi))$ (Monotonicity);

- $P(P(\varphi) \oplus P(\psi)) \leftrightarrow P(\varphi) \oplus P(\psi)$ (Idempotency);

- $P(\varphi \oplus \psi) \leftrightarrow[P(\varphi) \oplus P(\psi \ominus(\varphi \odot \psi))]$ (Additivity);

- from $\phi$ derive $P(\phi)$ (Necessitation).

Proofs are defined with no modification from the classical definition and $\vdash_{S F P} \phi$ will be used to denote that $\phi$ is a theorem of $\operatorname{SFP}(\mathrm{L}, \mathrm{E})$. If $\Gamma \cup\{\phi\}$ is a subset of SPFm, we will write $\Gamma \vdash_{S F P} \phi$ if there is a proof of $\phi$ from $\Gamma$.

The logic $\operatorname{SFP}(\mathrm{E}, \mathrm{E})$ is algebraizable and its equivalent algebraic semantics is the variety $\mathbb{S M V}$ of $M V$-algebras with internal state (or $S M V$-algebras for short).

Definition 2 ([6]). An $S M V$-algebra is a pair $(\mathbf{A}, \sigma)$ where $\mathbf{A}$ is an $M V$-algebra and the internal state $\sigma: A \rightarrow A$ satisfies the following equations:

$(\sigma 1) \sigma(\perp)=\perp$;

$(\sigma 2) \sigma(x \rightarrow y) \leq \sigma(x) \rightarrow \sigma(y)$

$(\sigma 3) \sigma(\sigma(x \oplus y)))=\sigma(x \oplus y)$;

$(\sigma 5) \sigma(x \oplus y)=\sigma(x) \oplus \sigma(y \ominus(x \odot y))$.

Example 2. (1) Every idempotent endomorphism $\sigma$ of an MV-algebra A makes $(\mathbf{A}, \sigma)$ an SMV-algebra.

(2) Let $\mathcal{F}_{\mathbb{R}}(\omega)$ be the set of all continuous and piecewise linear functions with real coefficients. The pointwise application of $\oplus$ and $\neg$ of $[0,1]_{M V}$ makes $\mathcal{F}_{\mathbb{R}}(\omega)$ into an MV-algebra which contains all constant functions $\bar{\alpha}$ for every $\alpha \in[0,1]$. These functions are continuous and hence Riemann-integrable. Let $\sigma: \mathcal{F}_{\mathbb{R}}(\omega) \rightarrow \mathcal{F}_{\mathbb{R}}(\omega)$ be defined as follows: for every $f \in \mathcal{F}_{\mathbb{R}}(\omega)$ with $k$ variables,

$$
\sigma(f)=\overline{\int_{[0,1]^{k}} f \mathrm{~d} x .}
$$

That is, $\sigma(f)$ is the function which is constantly equal to the Riemann integral of $f$. This map $\sigma$ is an internal state of $\mathcal{F}_{\mathbb{R}}(\omega)$.

Proposition 3 ([6]). For every SMV-algebra $(\mathbf{A}, \sigma)$ the following properties hold:

(1) the image $\sigma(A)$ of $A$ under $\sigma$, endowed with the operations inherited from $\mathbf{A}$ forms an $M V$-subalgebra $\sigma(\mathbf{A})$ of $\mathbf{A}$;

(2) if $(\mathbf{A}, \sigma)$ is a subdirectly irreducible, then $\sigma(\mathbf{A})$ is totally ordered. As a consequence the variety $\mathbb{S M V}$ is generated by its elements $(\mathbf{A}, \sigma)$ such that $\sigma(\mathbf{A})$ is an $M V$-chain. 
Valuations to an SMV-algebra $(\mathbf{A}, \sigma)$ are defined in the usual way. A valuation $v$ is said to be a model of a formula $\phi$, if $v(\phi)=T$. A formula $\phi \in \mathbf{S P F m}$ is said to be an $S M V$-tautology $\left(\models_{S M V} \phi\right.$ in symbols), if $v(\phi)=\top$ for every SMValgebra and every valuation to it. Further, for every (finite or infinite) subset $\Gamma \cup\{\phi\}$ of SPFm, we will write $\Gamma \models_{S M V} \phi$ if for every SMV-algebra $(\mathbf{A}, \sigma)$ and every valuation $v$ to $(\mathbf{A}, \sigma)$ which is a model of each formulas in $\Gamma$, then $v$ is a model of $\phi$ as well.

Proposition 4 ([6, Theorem 4.5]). For every (finite or infinite) subset $\Gamma \cup \phi$ of SPFm, $\Gamma \vdash_{\mathrm{SFP}} \phi$ iff $\Gamma \models_{S M V} \phi$.

We end this section with the following observation.

Remark 2. One of the most important properties for a t-norm based fuzzy logic, is completeness with respect to the class of its standard algebras. By "standard" we usually mean linearly ordered structures, order-embeddable into the real unit interval. Although $\mathrm{SFP}(\mathrm{E}, \mathrm{E})$ is grounded on Eukasiewicz calculus which enjoys a standard completeness theorem, $\mathrm{SFP}(\mathrm{E}, \mathrm{E})$ is not standard complete in the usual sense. In fact, for instance, the equation $\sigma(x \wedge y)=\sigma(x) \wedge \sigma(y)$ holds in every SMV-chain, but it does not hold in every SMV-algebra and in particular it does not hold in the algebra $\left(\mathcal{F}_{\mathbb{R}}(\omega), \sigma\right)$ of Example 2(2).

\section{Toward a standard completeness for $\operatorname{SFP}(\mathrm{E}, \mathrm{E})$}

In this section we will present a class of SMV-algebras that, in the light of Remark 2, it is reasonable to call standard. Our main result will show that, for a restricted, yet quite expressive, subset of SPFm, a formula is provable in $\mathrm{SFP}(\mathrm{E}, \mathrm{\textrm {E }})$ iff it is true in these standard algebras.

Definition 3. The set $\mathbf{P F m}$ of purely probabilistic formulas is the smallest subset of SPFm which contains all the modal formulas $P(\psi)$ (for $\psi$ a Eukasiewicz formula) and which is closed under the connectives of Lukasiewicz logic.

Notice that each formula of $\mathbf{P F m}$ is in the form $t\left[P\left(\psi_{1}\right), \ldots, P\left(\psi_{k}\right)\right]$ for $t$ being a(n MV-)term. Thus, for instance $P(\varphi) \rightarrow(P(\psi) \oplus P(\gamma)) \in \mathbf{P F m}$, but neither $P(\psi \rightarrow P(\varphi))$, nor $P(\psi) \rightarrow \gamma$ belong to PFm. It is also worth to point out that $\mathbf{P F m}$ forms the language for the weaker logic, with respect to $\mathrm{SFP}(\mathrm{E}, \mathrm{E})$, introduced in [3] and denoted by $\mathrm{FP}(\mathrm{L}, \mathrm{\textrm {L }})$.

First of all, we will recall the definition of a class of SMV-algebras which was introduced in [7].

Definition 4. An $S M V$-algebra $(\mathbf{A}, \sigma)$ is said to be $\sigma$-simple if $\mathbf{A}$ is semisimple and $\sigma(\mathbf{A})$ is simple.

In the light of the last comment of Subsection 2 a $\sigma$-simple SMV-algebra $(\mathbf{A}, \sigma)$ can be regarded as an algebra of continuous functions on a compact Hausdorff space endowed with a normalized, idempotent, finitely additive and real-valued internal state $\sigma$. 
The notions of valuation in a $\sigma$-simple SMV-algebra and tautology are as in Section 4 . We will write $=_{\sigma} \phi$ to denote that the formula $\phi$ is a tautology in this setting.

In the proof of the following result we will make use of the construction of $M V$-tensor product which was introduced by Mundici in [13] and heavily used in [6] to internalize a state of an MV-algebra. In what follows we will restrict the attention to those MV-algebras $[0,1]_{M V} \otimes \mathbf{A}$ defined by taking the tensor product of the standard MV-algebra $[0,1]_{M V}$ with an MV-algebra A. For the sake of the present paper, it is important to recall the following properties: (1) the generators of $[0,1]_{M V} \otimes \mathbf{A}$ are of the form $\alpha \otimes a$ for $\alpha \in[0,1]$ and $a \in A ;(2)$ the maps $a \in A \mapsto 1 \otimes a$ and $\alpha \mapsto \alpha \otimes T$ respectively are embeddings of $\mathbf{A}$ and $[0,1]_{M V}$ into $[0,1]_{M V} \otimes \mathbf{A} ;(3)$ the top element of $[0,1]_{M V} \otimes \mathbf{A}$ is $1 \otimes T$. By (2), $[0,1]_{M V} \otimes \mathbf{A}$ contains isomorphic copies of both $[0,1]_{M V}$ and $\mathbf{A} .^{2}$

Theorem 2. For every formula $\phi \in \mathbf{P F m}, \vdash_{\mathrm{SFP}} \phi$ iff $\models_{\sigma} \phi$.

Proof. The left-to-right direction follows from Proposition 4.

In order to prove the right-to-left direction, assume $\nvdash_{\text {SFP }} \phi$. Thus, from Proposition 4 there exists an SMV-algebra $(\mathbf{A}, \sigma)$ and a valuation $v$ in $A$ such that $v(\phi)<\top$. Without loss of generality, by Proposition 3 , we can assume $(\mathbf{A}, \sigma)$ to be subdirectly irreducible and hence $\sigma(\mathbf{A})$ linearly ordered. Since $\phi \in \mathbf{P F m}$, it will be in the form

$$
t\left[P\left(\psi_{1}\right), \ldots, P\left(\psi_{k}\right)\right]
$$

where $t$ is a term in the language of MV-algebras. Let us denote by $S_{\phi}$ the following subset of $A$ :

$$
S_{\phi}=\left\{v(\phi), v\left(P\left(\psi_{1}\right)\right), \ldots, v\left(P\left(\psi_{k}\right)\right)\right\}=\left\{v(\phi), \sigma\left(v\left(\psi_{1}\right)\right), \ldots, \sigma\left(v\left(\psi_{k}\right)\right)\right\} .
$$

Therefore $S_{\phi}$ is a finite subset of $\sigma(A)$ and since $\sigma(\mathbf{A})$ is an MV-chain, there is a partial embedding $h_{\phi}$ of $S_{\phi}$ into the standard MV-algebra $[0,1]_{M V}$ (see Lemma $1(1))$ :

$$
h_{\phi}: S_{\phi} \hookrightarrow^{p}[0,1]_{M V}
$$

By Lemma $1(2), h_{\phi}$ extends to a homomorphism, that we still denote by $h_{\phi}$, of the MV-subalgebra $\mathbf{S}=\left\langle S_{\phi}\right\rangle_{\sigma(\mathbf{A})}$ of $\sigma(\mathbf{A})$, generated by $S_{\phi}$, to $[0,1]_{M V}$ :

$$
h_{\phi}: \mathbf{S} \hookrightarrow[0,1]_{M V} .
$$

Notice that $h_{\phi}(v(\phi))<1$. By Remark $1, h_{\phi}$ extends to a state $s_{\phi}: \sigma(\mathbf{A}) \rightarrow[0,1]$. Thus, let $s: A \rightarrow[0,1]$ be the map

$$
s: a \in A \mapsto s_{\phi}(\sigma(a)) .
$$

Notice that $s$ and $s_{\phi}$ agree on $\sigma(A)$. Indeed by the idempotency of $\sigma$ (equation $(\sigma 3)$ of Definition 2), if $a=\sigma(b) \in \sigma(A), s(a)=s(\sigma(b))=s_{\phi}(\sigma \sigma(b))=$

${ }^{2}$ We invite the reader to consult [13] for an exhaustive description of the MV-tensor product construction and $[6,7]$ for its application to the theory of SMV-algebras. 
$s_{\phi}(\sigma(b))=s_{\phi}(a)$. Therefore, for every subformula $P\left(\psi_{i}\right)$ of $\phi$, since $s_{\phi}$ extends $h_{\phi}$,

$$
s\left(v\left(\psi_{i}\right)\right)=s_{\phi}\left(\sigma\left(v\left(\psi_{i}\right)\right)\right)=h_{\phi}\left(v\left(P\left(\psi_{i}\right)\right)\right),
$$

which leads to the following fact.

Fact $1 t^{[0,1]}\left[s\left(v\left(\psi_{1}\right)\right), \ldots, s\left(v\left(\psi_{k}\right)\right)\right]<1$.

As a matter of fact, by (1)

$$
\begin{aligned}
t^{[0,1]}\left[s\left(v\left(\psi_{1}\right)\right), \ldots, s\left(v\left(\psi_{k}\right)\right)\right] & =t^{[0,1]}\left[h_{\phi}\left(v\left(P\left(\psi_{1}\right)\right)\right), \ldots, h_{\phi}\left(v\left(P\left(\psi_{k}\right)\right)\right)\right] \\
& \left.=h_{\phi}\left(t^{\mathbf{A}}\left[v\left(P\left(\psi_{1}\right)\right)\right), \ldots, v\left(P\left(\psi_{k}\right)\right)\right]\right) \\
& =h_{\phi}\left(v\left(t\left[P\left(\psi_{1}\right), \ldots, P\left(\psi_{k}\right)\right]\right)\right) \\
& =h_{\phi}(v(\phi))<1 .
\end{aligned}
$$

In order to get the claim, we have now to define a $\sigma$-simple SMV-algebra $\left(\mathbf{M}, \sigma_{M}\right)$ and a valuation $w$ to $M$ such that $w(\phi)<1$. To this aim, let $\mathbf{N}=[0,1]_{M V} \otimes$ A and $s_{N}: N \rightarrow[0,1]$ be such that $s_{N}(\alpha \otimes a)=\alpha \cdot s(a)$. Than $s_{N}$ is a state of $\mathbf{N}$ which extends $s$ (indeed, $\mathbf{A}$ is a subalgebra of $\mathbf{N}$ ). Regardless of the semisimplicity of $\mathbf{A}, \mathbf{N}$ needs not to be semisimple (see [13, Theorem 3.3]). Thus, let $\mathbf{M}$ its most general semisimple quotient:

$$
\mathbf{M}=\mathbf{N} / \operatorname{Rad}(\mathbf{N}) \text { and } \sigma_{M}: m / \operatorname{Rad}(\mathbf{N}) \in M \mapsto s_{N}(m) .
$$

Notice that $\sigma_{M}$ is well-defined because of Proposition 1. Moreover, $\mathbf{M}$ is semisimple, $\sigma_{M}$ is an internal state of $\mathbf{M}$ (because the range $[0,1]_{M V}$ of $\sigma_{M}$ is a subalgebra of $\mathbf{M})$ and $\sigma_{M}(\mathbf{M})$ is clearly simple. Thus, $\left(\mathbf{M}, \sigma_{M}\right)$ is $\sigma$-simple.

Let $w$ be the following valuation to $M$ : for each propositional variable $q$, $w(q)=1 \otimes v(q)$ and $w(P(\gamma))=\sigma_{M}(v(\gamma)) \otimes \top$. Notice that, for every subformula $P\left(\psi_{i}\right)$ of $\phi$, one has

$$
w\left(P\left(\psi_{i}\right)\right)=\sigma_{M}\left(v\left(\psi_{i}\right)\right) \otimes \top=s_{N}\left(v\left(\psi_{i}\right)\right) \otimes \top=s\left(v\left(\psi_{i}\right)\right) \otimes \top,
$$

and hence, by Fact $1, w(\phi)=t^{[0,1]}\left[s\left(v\left(\psi_{1}\right)\right), \ldots, s\left(v\left(\psi_{k}\right)\right)\right] \otimes \top<1 \otimes \top$.

We now introduce a further refined semantics for $\operatorname{SFP}(\mathrm{E}, \mathrm{E})$. To this end let $\mathcal{F}_{\mathbb{R}}(\omega)$ be the MV-algebra of Example 2(2). It is worth to notice that $\mathcal{F}_{\mathbb{R}}(\omega)$ is semisimple [7, Proposition 2.4]. Further, every state $s: \mathcal{F}_{\mathbb{R}}(\omega) \rightarrow[0,1]$ can be easily internalized by considering the map $\sigma_{s}: \mathcal{F}_{\mathbb{R}}(\omega) \rightarrow \mathcal{F}_{\mathbb{R}}(\omega)$ defined as follows: for every $a \in \mathcal{F}_{\mathbb{R}}(\omega)$

$$
\sigma_{s}(a)=\overline{s(a)}
$$

Then $\left(\mathcal{F}_{\mathbb{R}}(\omega), \sigma_{s}\right)$ is a $\sigma$-simple SMV-algebra. Notice that, thanks to Theorem 1 the internal state $\sigma_{s}$ assigns to every $f \in \mathcal{F}_{\mathbb{R}}(\omega)$, the real number $\int f \mathrm{~d} \mu$ : the Lebesgue integral of $f$ by a regular, Borel probability measure.

Definition 5. A standard SMV-algebra is a pair $\left(\mathcal{F}_{\mathbb{R}}(\omega), \sigma_{s}\right)$ where $s: \mathcal{F}_{\mathbb{R}}(\omega) \rightarrow$ $[0,1]$ is a state and $\sigma_{s}$ is defined as in (2). 
Taking into account that the free $\omega$-generated MV-algebra of Example 1(2) is a subalgebra of $\mathcal{F}_{\mathbb{R}}(\omega)$ (see [7, Proposition 2.4]), a valuation $v$ of $\operatorname{SFP}(\mathrm{E}, \mathrm{E})$ formulas in $\left(\mathcal{F}_{\mathbb{R}}(\omega), \sigma_{s}\right)$ can be inductively defined as follows:

$-v(\psi)=f_{\psi}$ for $\psi$ a Eukasiewicz formula;

- $v$ commutes with Łukasiewicz connectives;

- $v(P(\psi))=\sigma_{s}(v(\psi))$.

We will write $\models_{S t} \phi$ to denote that $\phi$ is a tautology for this class of algebras.

Theorem 3. For every formula $\phi \in \mathbf{P F m}, \models_{\sigma} \phi$ iff $\models_{S t} \phi$. In other words, $\sigma$ simple $S M V$-algebras and standard SMV-algebras share the same $\mathbf{P F m - t a u t o l o g i e s . ~}$

Proof. Since every standard SMV-algebra is $\sigma$-simple, $\models_{\sigma} \phi$ implies $\models_{S t} \phi$. Let hence assume that $\nvdash_{\sigma} \phi$ and let $v$ be a valuation in a $\sigma$-simple SMV-algebra $(\mathbf{A}, \sigma)$ such that $v(\phi)<1$. Define $s: \mathcal{F}(\omega) \rightarrow[0,1]$ by the following stipulation: for every Lukasiewicz formula $\psi, s\left(f_{\psi}\right)=\sigma(v(\psi))$.

Clearly $s$ is a state of $\mathcal{F}(\omega)$ which, since $\mathcal{F}(\omega)$ is an MV-subalgebra of $\mathcal{F}_{\mathbb{R}}(\omega)$, extends to a state $\hat{s}$ of $\mathcal{F}_{\mathbb{R}}(\omega)$ by Proposition 2. Define $\sigma_{\hat{s}}$ by $(2)$ and the valuation $w$ on $\left(\mathcal{F}_{\mathbb{R}}(\omega), \sigma_{\hat{s}}\right)$ by $w(q)=f_{q}$. Let us prove that $w$ is not a model of $\phi$. Since $\phi \in \mathbf{P F m}$, it is in the form $t\left[P\left(\psi_{1}\right), \ldots, P\left(\psi_{k}\right)\right]$ and because $s$ and $\hat{s}$ agree on $\mathcal{F}(\omega)$, so do $\sigma_{s}$ and $\sigma_{\hat{s}}$ by definition. Thus, by (2) one has:

$$
\begin{aligned}
w(\phi) & =t^{[0,1]}\left[\sigma_{\hat{s}}\left(w\left(\psi_{1}\right)\right), \ldots, \sigma_{\hat{s}}\left(w\left(\psi_{k}\right)\right)\right] \\
& =t^{[0,1]}\left[\sigma_{\hat{s}}\left(f_{\psi_{1}}\right), \ldots, \sigma_{\hat{s}}\left(f_{\psi_{k}}\right)\right] \\
& =t^{[0,1]}\left[\sigma_{s}\left(f_{\psi_{1}}\right), \ldots, \sigma_{s}\left(f_{\psi_{k}}\right)\right] \\
& =t^{[0,1]}\left[s\left(f_{\psi_{1}}\right), \ldots, s\left(f_{\psi_{k}}\right)\right] \\
& =v(\phi)<1
\end{aligned}
$$

Therefore, $\forall_{S t} \phi$ which settles the claim.

The following result is a direct consequence of Theorem 2 and Theorem 3.

Corollary 1. For every formula $\phi \in \mathbf{P F m}, \vdash_{\mathrm{SFP}} \phi$ iff $\models_{S t} \phi$.

\section{Conclusion and future work}

In this paper we presented a partial solution to the problem of establishing a a standard completeness theorem for the probabilistic logic SFP $(\mathrm{L}, \mathrm{E})$ introduced in [6]. In particular we proved that, for a restricted class of formulas, theorems of $\mathrm{SFP}(\mathrm{E}, \mathrm{E})$ are tautologies for a class of SMV-algebras which are defined from realvalued metric spaces. In our future work on this argument we plan to extend the ideas and constructions which led to the proof of our main theorems to provide a standard completeness theorem for the whole language of $\operatorname{SFP}(\mathrm{E}, \mathrm{E})$.

\section{Acknowledgments}

The author acknowledges partial support by the Spanish Ramon y Cajal research program RYC-2016-19799; the Spanish FEDER/MINECO project TIN201571799-C2-1-P and the SYSMICS project (EU H2020-MSCA-RISE-2015, Project 689176). 


\section{References}

1. C. C. Chang. Algebraic Analysis of Many-valued Logics. Trans. Am. Math. Soc. 88, 467-490, 1958.

2. R. Cignoli, I. M. L. D'Ottaviano, D. Mundici. Algebraic Foundations of Many-valued Reasoning. Kluwer, 2000.

3. T. Flaminio, L. Godo. A logic for reasoning about the probability of fuzzy events. Fuzzy Sets and Systems 158(6): 625-638, 2007.

4. T. Flaminio, H. Hosni, S. Lapenta, Convex MV-algebras: Many-valued logics meet decision theory. Studia Logica 106(5): 913-945, 2018.

5. T. Flaminio, T. Kroupa. States of MV-algebras. Chapter XVII of Handbook of Mathematical Fuzzy Logic - volume 3, C. Fermüller, P. Cintula and C. Noguera (Eds.), Studies in Logic, Mathematical Logic and Foundations, vol. 58, College Publications, London, 2015.

6. T. Flaminio, F. Montagna. MV-algebras with internal states and probabilistic fuzzy logics. International Journal of Approximate Reasoning, 50(1): 138-152, 2009.

7. T. Flaminio, F. Montagna. Models for Many-Valued Probabilistic Reasoning. Journal of Logic and Computation, 21(3): 447-464, 2011.

8. P. Hájek. Metamathematics of Fuzzy Logics, Kluwer Academic Publishers, Dordrecht, 1998.

9. A. Horn, A. Tarski. Measures on Boolean algebras. Trans. Amer. Math. Soc., 64:467497, 1948.

10. T. Kroupa. Every state on semisimple MV-algebra is integral. Fuzzy Sets and Systems 157 (20), 2771-2787, 2006.

11. T. Kroupa. Representation and extension of states on MV-algebras. Archive for Mathematical Logic, 45: 381-392, 2006.

12. D. Mundici. Averaging the Truth-Value in Eukasiewicz Logic. Studia Logica 55: 113-127, 1995.

13. D. Mundici, Tensor Products and the Loomis-Sikorski Theorem for MV-Algebras. Advances in Applied Mathematics, 22: 227-248, 1999.

14. G. Panti. Invariant measures on free MV-algebras. Communications in Algebra, 36(8):2849-2861, 2009. 